\title{
Total and paired domination numbers of toroidal meshes *
}

\author{
$\mathrm{Fu}-\mathrm{Tao} \mathrm{Hu}$, Jun-Ming $\mathrm{Xu}^{\dagger}$ \\ Department of Mathematics \\ University of Science and Technology of China \\ Hefei, Anhui, 230026, China
}

\begin{abstract}
Let $G$ be a graph without isolated vertices. The total domination number of $G$ is the minimum number of vertices that can dominate all vertices in $G$, and the paired domination number of $G$ is the minimum number of vertices in a dominating set whose induced subgraph contains a perfect matching. This paper determines the total domination number and the paired domination number of the toroidal meshes, i.e., the Cartesian product of two cycles $C_{n}$ and $C_{m}$ for any $n \geq 3$ and $m \in\{3,4\}$, and gives some upper bounds for $n, m \geq 5$.
\end{abstract}

Keywords: combinatorics, total domination number, paired domination number, toroidal meshes, Cartesian product.

AMS Subject Classification: 05C25, 05C40, 05C12

\section{Introduction}

For notation and graph-theoretical terminology not defined here we follow [15]. Specifically, let $G=(V, E)$ be an undirected graph without loops, multi-edges and isolated vertices, where $V=V(G)$ is the vertex-set and $E=E(G)$ is the edge-set, which is a subset of $\{x y \mid x y$ is an unordered pair of $V\}$. A graph $G$ is nonempty if $E(G) \neq \emptyset$. Two vertices $x$ and $y$ are adjacent if $x y \in E(G)$. For a vertex $x$, denote $N(x)=\{y: x y \in E(G)\}$ be the neighborhood of $x$. For a subset $D \subseteq V(G)$, we use $G[D]$ to denote the subgraph of $G$ induced by $D$. We use $C_{n}$ and $P_{n}$ to denote a cycle and a path of order $n$, respectively, throughout this paper.

A subset $D \subseteq V(G)$ is called a dominating set if $N(x) \cap D \neq \emptyset$ for each vertex $x \in V(G) \backslash D$. The domination number $\gamma(G)$ is the minimum cardinality of a dominating set. A thorough study of domination appears in [6,7]. A subset $D \subseteq V(G)$ of $G$ is called a total dominating set, introduced by Cockayne et al. [2], if $N(x) \cap D \neq \emptyset$ for each vertex $x \in V(G)$ and the total domination number of $G$, denoted by $\gamma_{t}(G)$, is the minimum cardinality of a total dominating set of $G$. The total domination in graphs has been

*The work was supported by NNSF of China (No. 11071233).

${ }^{\dagger}$ Corresponding author: xujm@ustc.edu.cn 
extensively studied in the literature. A survey of selected recent results on this topic is given in [8] by Henning.

A dominating set $D$ of $G$ is called to be paired, introduced by Haynes and Slater [9, 10], if the induced subgraph $G[D]$ contains a perfect matching. The paired domination number of $G$, denoted by $\gamma_{p}(G)$, is the minimum cardinality of a paired dominating set of $G$. Clearly, $\gamma(G) \leq \gamma_{t}(G) \leq \gamma_{p}(G)$ since a paired dominating set is also a total dominating set of $G$, and $\gamma_{p}(G)$ is even. Pfaff, Laskar and Hedetniemi [12] and Haynes and Slater [10] showed that the problems determining the total-domination and the paired-domination for general graphs are NP-complete. Some exact values of totaldomination numbers and paired-domination numbers for some special classes of graphs have been determined by several authors. In particularly, $\gamma_{t}\left(P_{n} \times P_{m}\right)$ and $\gamma_{p}\left(P_{n} \times P_{m}\right)$ for $2 \leq m \leq 4$ are determined by Gravier [5], and Proffitt, Haynes and Slater [13], respectively.

Use $G_{n, m}$ to denote the toroidal meshes, i.e., the Cartesian product $C_{n} \times C_{m}$ of two cycles $C_{n}$ and $C_{m}$. Klavžar and Seifter [14] determined $\gamma\left(G_{n, m}\right)$ for any $n \geq 3$ and $m \in\{3,4,5\}$. In this paper, we obtain the following results.

$$
\begin{aligned}
& \gamma_{t}\left(G_{n, 3}\right)=\left\lceil\frac{4 n}{5}\right\rceil ; \\
& \gamma_{p}\left(G_{n, 3}\right)= \begin{cases}\left\lceil\frac{4 n}{5}\right\rceil & \text { if } n \equiv 0,2,4(\bmod 5), \\
\left\lceil\frac{4 n}{5}\right\rceil+1 & \text { if } n \equiv 1,3(\bmod 5) ;\end{cases} \\
& \gamma_{t}\left(G_{n, 4}\right)=\gamma_{p}\left(G_{n, 4}\right)= \begin{cases}n & \text { if } n \equiv 0(\bmod 4), \\
n+1 & \text { if } n \equiv 1,3(\bmod 4), \\
n+2 & \text { if } n \equiv 2(\bmod 4) .\end{cases}
\end{aligned}
$$

\section{Preliminary results}

In this section, we recall some definitions, notations and results used in the proofs of our main results. Throughout this paper, we assume that a cycle $C_{n}$ has the vertex-set $V\left(C_{n}\right)=\{1, \ldots, n\}$.

Use $G_{n, m}$ to denote the toroidal meshes, i.e., the Cartesian product $C_{n} \times C_{m}$, which is a graph with vertex-set $V\left(G_{n, m}\right)=\left\{x_{i j} \mid 1 \leq i \leq n, 1 \leq j \leq m\right\}$ and two vertices $x_{i j}$ and $x_{i^{\prime} j^{\prime}}$ being linked by an edge if and only if either $i=i^{\prime} \in V\left(C_{n}\right)$ and $j j^{\prime} \in E\left(C_{m}\right)$, or $j=j^{\prime} \in V\left(C_{m}\right)$ and $i i^{\prime} \in E\left(C_{n}\right)$.

Let $Y_{i}=\left\{x_{i j} \mid 1 \leq j \leq m\right\}$ for $1 \leq i \leq n$, called a set of vertical vertices in $G_{n, m}$.

In [4], Gavlas and Schultz defined an efficient total dominating set, which is such a total dominating set $D$ of $G$ that $|N(v) \cap D|=1$ for every $v \in V(G)$. The related research results can be found in [3, 4, 11].

Lemma 2.1 (Gavlas and Schult [4]) If a graph $G$ has an efficient total dominating set $D$, then the edge-set of the subgraph $G[D]$ forms a perfect matching, and so the cardinality of $D$ is even, and $\{N(v): v \in D\}$ partitions $V(G)$.

Lemma 2.2 Let $G$ be a k-regular graph of order $n$. Then $\gamma_{t}(G) \geq \frac{n}{k}$, with equality if and only if $G$ has an efficient total dominating set.

Proof. Since $G$ is $k$-regular, each $v \in V(G)$ can dominate at most $k$ vertices. Thus $\gamma_{t}(G) \geq \frac{n}{k}$. It is easy to observe that the equality holds if and only if there exists a total dominating set $D$ such that $\{N(v): v \in D\}$ partitions $V(G)$, equivalently, $D$ is an efficient total dominating set. 
Lemma $2.3 \gamma_{t}\left(G_{n, m}\right)=\gamma_{p}\left(G_{n, m}\right)=\frac{n m}{4}$ for $n, m \equiv 0(\bmod 4)$.

Proof. Let $D=\left\{x_{i j}, x_{i(j+1)}, x_{(i+2)(j+2)}, x_{(i+2)(j+3)}: i, j \equiv 1(\bmod 4)\right\}$, where $1 \leq i \leq n$ and $1 \leq j \leq m$. Figure 1 is such a set $D$ in $G_{8,4}$. It is easy to see that $D$ is a paired dominating set of $G_{n, m}$ with cardinality $\frac{n m}{4}$. Thus, $\gamma_{p}\left(G_{n, m}\right) \leq \frac{n m}{4}$.

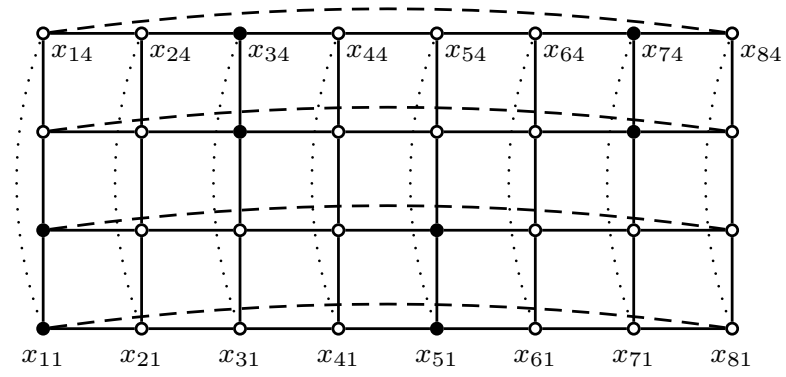

Figure 1: The minimum total (paired) dominating set (bold vertices) of $G_{8,4}$

By Lemma 2.2, $\gamma_{t}\left(G_{n, m}\right) \geq \frac{n m}{4}=n$. Since $\gamma_{t}\left(G_{n, m}\right) \leq \gamma_{p}\left(G_{n, m}\right), \gamma_{t}\left(G_{n, m}\right)=$ $\gamma_{p}\left(G_{n, m}\right)=\frac{n m}{4}$.

\section{Total and paired domination number of $G_{n, 3}$}

In this section, we determine the exact values of the total and the paired domination numbers of $G_{n, 3}$, which can be stated the following theorem.

Theorem 3.1 For any $n \geq 3$,

$$
\gamma_{t}\left(G_{n, 3}\right)=\left\lceil\frac{4 n}{5}\right\rceil
$$

and

$$
\gamma_{p}\left(G_{n, 3}\right)= \begin{cases}\left\lceil\frac{4 n}{5}\right\rceil, & \text { if } n \equiv 0,2,4(\bmod 5) ; \\ \left\lceil\frac{4 n}{5}\right\rceil+1, & \text { if } n \equiv 1,3(\bmod 5) .\end{cases}
$$

Proof. Let $D$ be a minimum total dominating set of $G_{n, 3}$. First, we may assume that $\left|Y_{i} \cap D\right| \leq 2$ for any $1 \leq i \leq n$. Indeed, if $\left|Y_{i} \cap D\right|=3$ for some $i \notin\{1, n\}$, then the set $D^{\prime}=\left(D \backslash\left\{x_{i 1}, x_{i 3}\right\}\right) \cup\left\{x_{(i-1) 2}, x_{(i+1) 2}\right\}$ is also a total dominating set of $G_{n, 3}$ with $\left|D^{\prime}\right|=|D|$.

Let $\alpha_{k}$ be the number of $i$ 's for which $\left|Y_{i} \cap D\right|=k$ for $1 \leq i \leq n$ and $0 \leq k \leq 2$. Then we have

$$
\alpha_{0}+\alpha_{1}+\alpha_{2}=n .
$$

Assume $\left|Y_{i} \cap D\right|=0$ for some $i \notin\{1, n\}$. At least one of $\left|Y_{i-1} \cap D\right|$ and $\left|Y_{i+1} \cap D\right|$ is 2 since the three vertices in $Y_{i}$ should be dominated by $D$, which means that

$$
2 \alpha_{2}-\alpha_{0} \geq 0 .
$$

If $\left|Y_{i} \cap D\right|=2$ for some $i$ with $1 \leq i \leq n$, then the two vertices in $Y_{i} \cap D$ can dominate at most 7 vertices. Since any vertex $x \in D$ can dominate at most 4 vertices, we have

$$
4 \alpha_{1}+7 \alpha_{2} \geq 3 n \text {. }
$$


The sum of (3.1), (3.2) and (3.3) implies

$$
5 \alpha_{1}+10 \alpha_{2} \geq 4 n,
$$

and, hence,

$$
\gamma_{t}\left(G_{n, 3}\right)=|D|=\alpha_{1}+2 \alpha_{2} \geq\left\lceil\frac{4 n}{5}\right\rceil .
$$

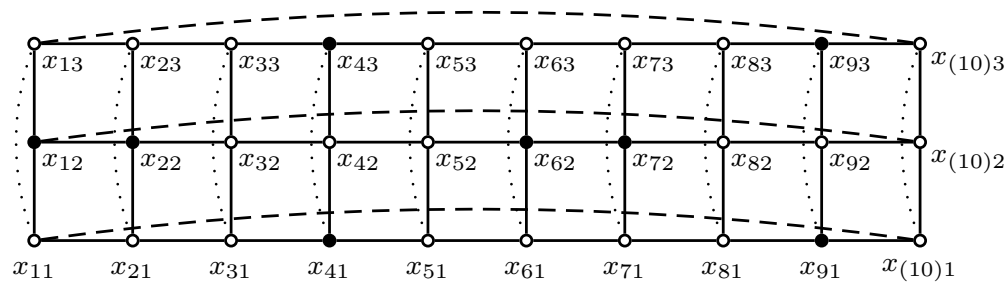

Figure 2: The minimum paired dominating set (bold vertices) of $G_{10,3}$

To obtain the upper bounds of $\gamma_{t}\left(G_{n, 3}\right)$ and $\gamma_{p}\left(G_{n, 3}\right)$, we set

$$
D=\left\{x_{i 2}: i \equiv 1,2(\bmod 5)\right\} \cup\left\{x_{j 1}, x_{j 3}: j \equiv 4(\bmod 5)\right\},
$$

where $1 \leq i \leq n$. See Figure 2, where $D$ consists of bold vertices.

If $n \not \equiv 3(\bmod 5)$, then $D$ is a total dominating set and $\gamma_{t}\left(G_{n, 3}\right) \leq|D|=\left\lceil\frac{4 n}{5}\right\rceil$.

If $n \equiv 3(\bmod 5)$, then $D \cup\left\{x_{n 2}\right\}$ is a total dominating set and $\gamma_{t}\left(G_{n, 3}\right) \leq|D|+1=$ $\left\lceil\frac{4 n}{5}\right\rceil$.

Combining these facts with (3.4), we have that $\gamma_{t}\left(G_{n, 3}\right)=\left\lceil\frac{4 n}{5}\right\rceil$.

If $n \equiv 0,2,4(\bmod 5)$, then $D$ is a paired dominating set and $\gamma_{p}\left(G_{n, 3}\right) \leq|D|=\left\lceil\frac{4 n}{5}\right\rceil$.

If $n \equiv 1(\bmod 5)$, then $D \cup\left\{x_{n 1}\right\}$ is a paired dominating set and $\gamma_{p}\left(G_{n, 3}\right) \leq|D|+1=$ $\left\lceil\frac{4 n}{5}\right\rceil+1$.

If $n \equiv 3(\bmod 5)$, then $D \cup\left\{x_{n 1}, x_{n 2}\right\}$ is a paired dominating set and $\gamma_{p}\left(G_{n, 3}\right) \leq$ $|D|+2=\left\lceil\frac{4 n}{5}\right\rceil+1$.

Since $\gamma_{p}\left(G_{n, 3}\right) \geq \gamma_{t}\left(G_{n, 3}\right)$ and $\gamma_{p}\left(G_{n, 3}\right)$ is even, $\gamma_{p}\left(G_{n, 3}\right)=\left\lceil\frac{4 n}{5}\right\rceil$ if $n \equiv 0,2,4(\bmod 5)$, and $\gamma_{p}\left(G_{n, 3}\right)=\left\lceil\frac{4 n}{5}\right\rceil+1$ if $n \equiv 1,3(\bmod 5)$.

The theorem follows.

\section{Total and paired domination number of $G_{n, 4}$}

In this section, we determine the exact values of $\gamma_{t}\left(G_{n, 4}\right)$ and $\gamma_{p}\left(G_{n, 4}\right)$, the latter has been announced by Brešar, Henning and Rall [1], but without proofs.

Lemma $4.1 \gamma_{p}\left(G_{n, 4}\right)=\gamma_{t}\left(G_{n, 4}\right)=n+1$ for $n \equiv 1,3(\bmod 4)$.

Proof. For $n \equiv 1(\bmod 4)$, let

$$
D=\left\{x_{i 1}, x_{i 2}, x_{(i+2) 3}, x_{(i+2) 4}: i \equiv 1(\bmod 4), i \neq n\right\} \cup\left\{x_{n 1}, x_{n 2}\right\} .
$$


Then $D$ is a paired dominating set of $G_{n, 4}$ with cardinality $n+1$. For $n \equiv 3(\bmod 4)$, $D=\left\{x_{i 1}, x_{i 2}, x_{(i+2) 3}, x_{(i+2) 4}: i \equiv 1(\bmod 4)\right\}$ is a paired dominating set of $G_{n, 4}$ with cardinality $n+1$. Thus, $\gamma_{t}\left(G_{n, 4}\right) \leq \gamma_{p}\left(G_{n, 4}\right) \leq n+1$ for $n \equiv 1,3(\bmod 4)$.

By Lemma 2.2, $\gamma_{t}\left(G_{n, 4}\right) \geq \frac{4 n}{4}=n$. Now, we prove $\gamma_{t}\left(G_{n, 4}\right) \geq n+1$. Suppose to the contrary that $\gamma_{t}\left(G_{n, 4}\right)=n$. By Lemma 2.2, $G_{n, 4}$ has an efficient total dominating set $D^{\prime}$. By Lemma 2.1, $\left|D^{\prime}\right|=n$ is even, a contradiction. Therefore $\gamma_{t}\left(G_{n, 4}\right)>n$, and hence $\gamma_{p}\left(G_{n, 4}\right)=\gamma_{t}\left(G_{n, 4}\right)=n+1$.

Lemma $4.2 \gamma_{t}\left(G_{n, 4}\right) \leq \gamma_{p}\left(G_{n, 4}\right) \leq n+2$ for $n \equiv 2(\bmod 4)$.

Proof. Let

$$
D=\left\{x_{i 1}, x_{i 2}, x_{(i+2) 3}, x_{(i+2) 4}: i \equiv 1(\bmod 4), i \leq n-2\right\} \cup\left\{x_{(n-1) 1}, x_{(n-1) 2}, x_{n 1}, x_{n 2}\right\} .
$$

Then $D$ is a paired dominating set of $G_{n, 4}$ with cardinality $n+2$. Thus, $\gamma_{t}\left(G_{n, 4}\right) \leq$ $\gamma_{p}\left(G_{n, 4}\right) \leq n+2$.

To prove $\gamma_{t}\left(G_{n, 4}\right) \geq n+2$ for $n \equiv 2(\bmod 4)$, we need the following notations and two lemmas. Let $H_{i}^{j}=Y_{i} \cup Y_{i+1} \cup \ldots \cup Y_{i+j-1}$, and let $G_{i}^{j}$ be the graph obtained from $G_{n, 4}-H_{i}^{j}$ by adding the edge-set $\left\{x_{(i-1) k} x_{(i+j) k}: 1 \leq k \leq 4\right\}$, where the subscripts are modulo $n$. Clearly, $G_{i}^{j} \cong G_{n-j, 4}$.

Lemma 4.3 Let $D$ be a total dominating set of $G_{n, 4}$. Then $\left|D \cap H_{i}^{4}\right| \geq 4$ for any $i$ with $1 \leq i \leq n$. Moreover, if there exists some $i$ with $1 \leq i \leq n$ such that $|N(v) \cap D|=1$ for any vertex $v$ in $H_{i}^{4}$, then $D^{\prime}=D \backslash\left(D \cap H_{i}^{4}\right)$ is a total dominating set of $G_{i}^{4}$.

Proof. Without loss of generality, assume $i=2$. It can be easy verified to dominate 8 vertices in $Y_{3} \cup Y_{4}$, at least 4 vertices are needed, and hence $\left|D \cap H_{2}^{4}\right| \geq 4$.

We now show the second assertion. Suppose to the contrary that $D^{\prime}$ is not a total dominating set of $G_{2}^{4}$. Then there is a vertex $u$ in $Y_{1} \cup Y_{6}$ such that it is not dominated by $D^{\prime}$, that is, $N_{G_{2}^{4}}(u) \cap D^{\prime}=\emptyset$. Without loss of generality assume $u=x_{11}$. Then $x_{21} \in D$ and $x_{61} \notin D$. Also $x_{41} \notin D$ since $\left|N\left(x_{31}\right) \cap D\right|=1$.

Since $x_{33}$ should be dominated by $D$ and $\left|N\left(x_{33}\right) \cap D\right|=1$, only one of $x_{32}, x_{34}, x_{23}$, and $x_{43}$ belongs to $D$. If $x_{32} \in D$ or $x_{34} \in D$, then $\left|N\left(x_{31}\right) \cap D\right| \geq 2$, a contradiction. If $x_{23} \in D$, then $\left|N\left(x_{22}\right) \cap D\right| \geq 2$, a contradiction. Thus, $x_{43} \in D$. Since $x_{51}$ should be dominated by $D, x_{52} \in D$ or $x_{54} \in D$. But then $\left|N\left(x_{53}\right) \cap D\right| \geq 2$, a contradiction. Thus, $D^{\prime}=D \backslash\left(D \cap H_{2}^{4}\right)$ is a total dominating set of $G_{i}^{4}$.

Lemma 4.4 Let $D$ be a total dominating set of $G_{n, 4}$. If $x_{i j}$ is dominated by two vertices $u, v \in D$, then there exists a vertex $w$ in $H_{i-1}^{2}$ or $H_{i}^{2}$ such that $|N(w) \cap D| \geq 2$.

Proof. Without loss of generality, let $i=j=2$. If $u, v \in Y_{2}$, then assume $u=x_{21}$, $v=x_{23}$ and, hence, $\left|N\left(x_{24}\right) \cap D\right| \geq 2$.

If one of $u$ and $v$ is in $Y_{2}$ and another is in $Y_{1} \cup Y_{3}$, then without loss of generality assume $u=x_{21} \in Y_{2}$ and $v=x_{32} \in Y_{3}$. And then $\left|N\left(x_{31}\right) \cap D\right| \geq 2$.

If one of $u$ and $v$ is in $Y_{1}$ and another is in $Y_{3}$, then without loss of generality assume $u=x_{12} \in Y_{2}$ and $v=x_{32} \in Y_{3}$. Since $x_{24}$ should be dominated by $D$, let $s \in N\left(x_{24}\right) \cap D$. It is clearly that $N(s) \cap N(u) \neq \emptyset$ or $N(s) \cap N(v) \neq \emptyset$, which implies that there exists a vertex $w \notin\{u, v\}$ in $H_{1}^{2} \cup H_{2}^{2}$ such that $|N(w) \cap D| \geq 2$. 
Lemma $4.5 \gamma_{t}\left(G_{n, 4}\right)=\gamma_{p}\left(G_{n, 4}\right)=n+2$ for $n \equiv 2(\bmod 4)$.

Proof. By Lemma 4.2, we only need to show $\gamma_{t}\left(G_{n, 4}\right) \geq n+2$. To this end, let $n=4 k+2$. We proceed by induction on $k \geq 1$. It is easy to verify that $\gamma_{t}\left(G_{6,4}\right)=8$ and $\gamma_{t}\left(G_{10,4}\right)=12$. The conclusion is true for $k=1,2$. Assume that the induction hypothesis is true for $k-1$ with $k \geq 3$.

Let $D$ be a minimum total dominating set of $G_{n, 4}$, where $n=4 k+2$ for $k \geq 3$. Assume to the contrary that $|D| \leq n+1$. Since any vertex $u$ can dominate at most 4 vertices in $G_{n, 4}$ and $\left|V\left(G_{n, 4}\right)\right|=4 n$, there are at most four vertices such that each of them is dominated by at least two vertices in $D$.

We now prove that there exists some $i \in\{1,2, \ldots, n\}$ such that $|N(v) \cap D|=1$ for any vertex $v \in H_{i}^{4}$. There is nothing to do if there are at most three vertices such that each of them is dominated by at least two vertices since $n \geq 14$. Now, assume there are exactly four vertices such that each of them is dominated by at least two vertices. By Lemma 4.4, there exists two integers $s$ and $t$ with $1 \leq s, t \leq n$ such that two of the four vertices are in $H_{s}^{2}$ and the other two are in $H_{t}^{2}$. Therefore, there exists an integer $i$ with $1 \leq i \leq n$ such that for any vertex $v \in Y_{i},|N(v) \cap D|=1$ since $n \geq 14$.

By Lemma 4.3. $\left|D \cap H_{i}^{4}\right| \geq 4$ and $D^{\prime}=D \backslash\left(D \cap H_{i}^{4}\right)$ is a total dominating set of $G_{i}^{4} \cong G_{n-4,4}$. By the inductive hypothesis, $\left|D^{\prime}\right| \geq \gamma_{t}\left(G_{n-4,4}\right) \geq n-2$. It follows that

$$
n+1 \geq|D|=\left|D \cap H_{i}^{4}\right|+\left|D^{\prime}\right| \geq 4+n-2=n+2,
$$

a contradiction, which implies that $\gamma_{t}\left(G_{n, 4}\right)=|D| \geq n+2$. By the induction principle, the lemma follows.

We state the above results as the following theorem.

Theorem 4.1 For any integer $n \geq 3$,

$$
\gamma_{t}\left(G_{n, 4}\right)=\gamma_{p}\left(G_{n, 4}\right)= \begin{cases}n, & \text { if } n \equiv 0(\bmod 4) \\ n+1, & \text { if } n \equiv 1,3(\bmod 4) \\ n+2, & \text { if } n \equiv 2(\bmod 4)\end{cases}
$$

\section{$5 \quad$ Upper bounds of $\gamma_{p}\left(G_{n, m}\right)$ for $n, m \geq 5$}

The values of $\gamma_{t}\left(G_{n, m}\right)$ and $\gamma_{p}\left(G_{n, m}\right)$ for $m \in\{3,4\}$ have been determined in the above sections, but their values for $m \geq 5$ have been not determined yet. In this section, we present their upper bounds. Since $\gamma_{t}(G) \leq \gamma_{p}(G)$ for any graph $G$ without isolated vertices, we establish upper bounds only for $\gamma_{p}\left(G_{n, m}\right)$ if we can not obtain a smaller upper bound of $\gamma_{t}\left(G_{n, m}\right)$ than that of $\gamma_{p}\left(G_{n, m}\right)$.

Lemma $5.1 \gamma_{t}\left(G_{n, m}\right) \leq \gamma_{t}\left(G_{n+1, m}\right)$ and $\gamma_{p}\left(G_{n, m}\right) \leq \gamma_{p}\left(G_{n+1, m}\right)$.

Proof. Let $D$ be a minimum paired (total) dominating set of $G_{n+1, m}$.

If $D \cap Y_{n+1}=\emptyset$, then $D$ is also a paired (total) dominating set of $G_{n, m}$, and hence $\gamma_{p}\left(G_{n, m}\right) \leq|D|\left(\gamma_{t}\left(G_{n, m}\right) \leq|D|\right)$.

Assume $D \cap Y_{n+1} \neq \emptyset$ below. Let $A=\left\{j \mid x_{(n+1) j} \in D\right\}$ and $B=\left\{j \mid x_{n j} \in D\right\}$. Then $D^{\prime}=\left(D \backslash Y_{n+1}\right) \cup\left\{x_{(n-1) j} \mid j \in A \cap B\right\} \cup\left\{x_{n j} \mid j \in A \backslash B\right\}$ is a total dominating set of $G_{n, m}$ and $\left|D^{\prime}\right| \leq|D|$. Therefore $\gamma_{t}\left(G_{n, m}\right) \leq \gamma_{t}\left(G_{n+1, m}\right)$. 
The vertex set $D^{\prime}$ may not be a paired dominating set of $G_{n, m}$, that means, the induced subgraph $G$ by $D^{\prime}$ in $G_{n, m}$ may contains odd connected components. Let $p$ be the number of odd connected components in $G$. It is clear that $\left|D^{\prime}\right| \leq|D|-p$ by the construction of $D^{\prime}$ from $D$. Therefore, we can obtain $D^{\prime \prime}$ by adding at most $p$ vertices to $D^{\prime}$ such that the induced subgraph by $D^{\prime \prime}$ in $G_{n, m}$ does not contain odd connected components. Then $D^{\prime \prime}$ is a paired dominating set of $G_{n, m}$, and hence $\gamma_{p}\left(G_{n, m}\right) \leq\left|D^{\prime \prime}\right| \leq$ $|D|$.

Theorem $5.1 \gamma_{p}\left(G_{n, m}\right) \leq 4\left\lceil\frac{n}{4}\right\rceil\left\lceil\frac{m}{4}\right\rceil$.

Proof. Let $n=4 a-i$ and $m=4 b-j$ where $0 \leq i, j \leq 3$. By Lemma 2.3, $\gamma_{p}\left(G_{4 a, 4 b}\right)=$ $4 a b=4\left\lceil\frac{n}{4}\right\rceil\left\lceil\frac{m}{4}\right\rceil$. By Lemma 5.1, $\gamma_{p}\left(G_{m, n}\right) \leq \gamma_{p}\left(G_{4 a, 4 b}\right)=4\left\lceil\frac{n}{4}\right\rceil\left\lceil\frac{m}{4}\right\rceil$.

For $n, m \geq 5$, let $m \equiv a(\bmod 4)$ and $n \equiv b(\bmod 4)$ where $0 \leq a, b \leq 3$. We will establish some better bounds of $\gamma_{t}\left(G_{n, m}\right)$ and $\gamma_{p}\left(G_{n, m}\right)$ than those in Theorem 5.1 for some special $a$ and $b$. Without loss of generality, we can assume $b \geq a$ since $G_{n, m} \cong G_{m, n}$. Let

$$
D_{e}=\left\{x_{i j}, x_{i(j+1)}, x_{(i+2)(j+2)}, x_{(i+2)(j+3)}: i, j \equiv 1(\bmod 4)\right\},
$$

where $1 \leq i \leq n-2,1 \leq j \leq m-2$, and $n, m \geq 5$.

Theorem $5.2 \gamma_{p}\left(G_{n, m}\right) \leq \frac{(n+1) m}{4}$ for $m \equiv 0(\bmod 4)$ and $n \equiv 1(\bmod 4)$.

Proof. Let $D=D_{e} \cup\left\{x_{n j}, x_{n(j+1)}: j \equiv 1(\bmod 4)\right\}$, where $1 \leq j \leq m-2$. Then, it is easy to see that $D$ is a paired dominating set of $G_{n, m}$ with cardinality $\frac{(n+1) m}{4}$. Thus, $\gamma_{p}\left(G_{n, m}\right) \leq \frac{(n+1) m}{4}$.

Theorem $5.3 \gamma_{t}\left(G_{n, m}\right) \leq \frac{(n+1)(m+1)}{4}$ and $\gamma_{p}\left(G_{n, m}\right) \leq \frac{(n+1)(m+1)}{4}+1$ for $m, n \equiv 1(\bmod 4)$.

Proof. Let $D=D_{e} \cup\left\{x_{n j}, x_{n(j+1)}, x_{(i+1)(m-1)}, x_{(i+2) m}: i, j \equiv 1(\bmod 4)\right\} \cup\left\{x_{n m}\right\}$, where $1 \leq i \leq n-2$ and $1 \leq j \leq m-2$. Then, it is easy to see that $D$ is a total dominating set of $G_{n, m}$ with cardinality $\frac{(n+1)(m+1)}{4}$, and $D \cup\left\{x_{n(m-1)}\right\}$ is a paired dominating set of $G_{n, m}$ with cardinality $\frac{(n+1)(m+1)}{4}+1$. Thus, $\gamma_{t}\left(G_{n, m}\right) \leq \frac{(n+1)(m+1)}{4}$ and $\gamma_{p}\left(G_{n, m}\right) \leq$ $\frac{(n+1)(m+1)}{4}+1$.

Theorem $5.4 \gamma_{t}\left(G_{n, m}\right) \leq \frac{(n+1)(m+1)}{4}-3$ and $\gamma_{p}\left(G_{n, m}\right) \leq \frac{(n+1)(m+1)}{4}-2$ for $m \equiv$ $1(\bmod 4)$ and $n \equiv 3(\bmod 4)$.

Proof. Let $D=\left(D_{e} \cup\left\{x_{(i+1)(m-1)}, x_{(i+2) m}: i \equiv 1(\bmod 4)\right\}\right) \backslash\left\{x_{n(m-2)}, x_{n m}\right\}$, where $1 \leq i \leq n-2$. Then, $D$ is a paired dominating set of $G_{n, m}$ with cardinality $\frac{(n+1)(m+1)}{4}-2$, and $D \backslash\left\{x_{2(m-1)}\right\}$ is a total dominating set of $G_{n, m}$ with cardinality $\frac{(n+1)(m+1)}{4}-3$. Thus, $\gamma_{t}\left(G_{n, m}\right) \leq \frac{(n+1)(m+1)}{4}-3$ and $\gamma_{p}\left(G_{n, m}\right) \leq \frac{(n+1)(m+1)}{4}-2$.

Corollary $5.1 \gamma_{t}\left(G_{n, m}\right) \leq \frac{(n+2)(m+1)}{4}-3$ and $\gamma_{p}\left(G_{n, m}\right) \leq \frac{(n+2)(m+1)}{4}-2$ for $m \equiv$ $1(\bmod 4)$ and $n \equiv 2(\bmod 4)$.

Proof. By Lemma 5.1, $\gamma_{t}\left(G_{n, m}\right) \leq \gamma_{t}\left(G_{n+1, m}\right)$ and $\gamma_{p}\left(G_{n, m}\right) \leq \gamma_{p}\left(G_{n+1, m}\right)$. The corollary follows from Theorem 5.4.

Theorem $5.5 \gamma_{p}\left(G_{n, m}\right) \leq \frac{(n+2)(m+2)}{4}-6$ for $m, n \equiv 2(\bmod 4)$.

Proof. Let $D=\left(D_{e} \cup\left\{x_{i(m-2)}, x_{i(m-1)}, x_{(i+2)(m-1)}, x_{(i+2) m}: i \equiv 1(\bmod 4)\right\} \cup\left\{x_{(n-1) j}\right.\right.$, $\left.\left.x_{(n-1)(j+1)}, x_{n(j+2)}, x_{n(j+3)}: j \equiv 1(\bmod 4)\right\} \cup\left\{x_{n(m-1)}\right\}\right) \backslash\left\{x_{1(m-2)}, x_{1(m-1)}, x_{n(m-3)}\right\}$, where $1 \leq i \leq n-2$ and $1 \leq j \leq m-2$. Then $D$ is a paired dominating set of $G_{n, m}$ with cardinality $\frac{(n+2)(m+2)}{4}-6$. Thus, $\gamma_{p}\left(G_{n, m}\right) \leq \frac{(n+2)(m+2)}{4}-6$. 


\section{References}

[1] B. Brešar, M. A. Henning, and D. F. Rall, Paired-domination of Cartesian products of graphs and rainbow domination. Electronic Notes in Discrete Mathematics, 22 (2005), 233-237.

[2] E. J. Cockayne, R. M. Dawes, S. T. Hedetniemi, Total domination in graphs. Networks, 10 (1980), 211-219.

[3] I. J. Dejter and O. Serra, Efficient dominating sets in Cayley graphs. Discrete Applied Mathematics, 129 (2003), 319-328.

[4] H. Gavlas and K. Schultz, Efficient open domination. Electronic Notes in Discrete Mathematics, 11 (2002), 681-691.

[5] S. Gravier, Total domination number of grid graphs. Discrete Applied Mathematics, 121 (2002), 119-128.

[6] T. W. Haynes, S. T. Hedetniemi, P. J. Slater, Fundamentals of Domination in Graphs. Marcel Dekker, New York, 1998.

[7] T. W. Haynes, S. T. Hedetniemi, P. J. Slater (Eds.), Domination in Graphs: Advanced Topics. Marcel Dekker, New York, 1998.

[8] M. A. Henning, A survey of selected recent results on total domination in graphs. Discrete Mathematics, 309(1) (2009), 32-63.

[9] T. W. Haynes and P. J. Slater, Paired-domination and the paired-domatic number. Congresses Numerantium, 109 (1995), 65-72.

[10] T. W. Haynes and P. J. Slater, Paired-domination in graphs. Networks, 32 (1998), 199-206.

[11] J. Huang and J.-M. Xu, The bondage numbers and efficient dominations of vertextransitive graphs. Discrete Mathematics, 308(4) (2008), 571-582.

[12] J. Pfaff, R.C. Laskar, S.T. Hedetniemi, NP-completeness of total and connected domination and irredundance for bipartite graphs, Technical Report 428, Clemson University, Dept. Math. Sciences, 1983.

[13] K. E. Proffitt, T. W. Haynes, and P. J. Slater, Paired-domination in grid graphs. Congresses Numerantium, 150 (2001), 161-172.

[14] S. Klavžar and N. Seifter, Dominating cartesian products of cycles. Discrete Applied Mathematics, 59 (1995), 129-136.

[15] J.-M. Xu, Theory and Application of Graphs. Kluwer Academic Publishers, Dordrecht/Boston/London, 2003. 